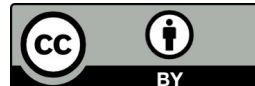

\title{
Sistema de referencia y contrarreferencia en el manejo de las emergencias obstétricas en el Hospital Rural de Lamas
}

Referral and counter-referral system in the management of obstetric emergencies in the Rural Hospital of Lamas

Sistema de referência e contra-referência na gestão de emergências obstétricas no Hospital Rural de Lamas

\section{ARTÍCULO GENERAL}

\author{
Rosa Pryscilia Cardenas Urrelo \\ https://orcid.org/0000-0003-4383-9765 \\ rpcardenasu@unsm.edu.pe
}

Universidad Nacional Mayor de San Marcos, Lima - Perú

Recibido 07 de Abril 2021 | Arbitrado y aceptado 19 de Junio 2021 | Publicado en 03 Setiembre 2021

\section{RESUMEN}

Se tuvo el objetivo de determinar el nivel de cumplimiento de la norma técnica de referencias y contrarreferencias de las emergencias obstétricas en el Hospital Rural de Lamas. Para ello, el estudio fue de tipo no experimental, con un diseño de estudio descriptivo simple, la muestra estuvo conformada por los responsables de las referencias a las gestantes, desde las cabeceras de micro redes de salud al Hospital Rural de Lamas, constituida por 69 referencias obstétricas que fueron atendidas y referidas en el en el periodo 2018, el instrumento empleado fue el cuestionario y la ficha de recolección de datos. El resultado destacó el nivel de manejo administrativo con un nivel "Regular" de 33\%, el nivel de manejo asistencial con un nivel "Regular" de $34 \%$, los diagnósticos más frecuentes con que son referidas las mujeres embarazadas es el "Aborto incompleto" y la hipertensión severa inducida por el embrazo; asimismo, las referencia son realizadas por el profesional Obstetra en un 39\%, seguido del personal médico con un $25 \%$. Por todo ello este artículo pudo concluir que el nivel de cumplimiento de la norma técnica de referencias y contrarreferencias de las emergencias obstétricas en el Hospital Rural de Lamas tiene un nivel "Regular" con un $34 \%$.

Palabras clave: Referencia, contrarreferencia, riesgo, atención y gestación

\section{ABSTRACT}

The objective was to determine the level of compliance with the technical standard of referrals and counterreferences of obstetric emergencies in the Rural Hospital of Lamas. For this, the study was non-experimental, with a simple descriptive study design, the sample was made up of those responsible for referrals to pregnant women, from the heads of micro health networks to the Rural Hospital of Lamas, consisting of 69 obstetric references that were attended and referred in the 2018 period, the instrument used was the questionnaire and the data collection sheet. The result highlighted the level of administrative management with a "Regular" level of $33 \%$, the level of care management with a "Regular" level of $34 \%$, the most frequent diagnoses with which pregnant women are referred is "Incomplete abortion" and severe pregnancy-induced hypertension; Likewise, referrals are made by the Obstetrician professional in $39 \%$, followed by medical personnel with $25 \%$. Therefore, this article was able to conclude that the level of compliance with the technical standard of referrals and counterreferences of obstetric emergencies in the Rural Hospital of Lamas has a "Regular" level with 34\%.

Keywords: Referral, counter referral, risk, care and pregnancy

\section{RESUMO}

O objetivo foi determinar o grau de cumprimento da norma técnica de encaminhamentos e contrarreferências de emergências obstétricas no Hospital Rural de Lamas. Para tanto, o estudo foi não experimental, com desenho de estudo descritivo simples, a amostra foi constituída pelos responsáveis pelos encaminhamentos às gestantes, desde os chefes das micro redes de saúde ao Hospital Rural de Lamas, constituída por 69 referenciais obstétricos. atendidos e encaminhados no período de 2018, o instrumento utilizado foi o questionário e a ficha de coleta de dados. $\mathrm{O}$ resultado destacou o nível de gestão administrativa com nível "Regular" de 33\%, o nível de gestão do cuidado com nível "Regular" de $34 \%$, os diagnósticos mais frequentes para os quais as gestantes são encaminhadas é "Aborto incompleto" e grave hipertensão induzida pela gravidez; Da mesma forma, os encaminhamentos são feitos pelo profissional obstetra com $39 \%$, seguido do pessoal médico com $25 \%$. Assim, este artigo permitiu concluir que o nível de cumprimento da norma técnica de encaminhamentos e contrarreferências de urgências obstétricas no Hospital Rural de Lamas apresenta um nível "Regular" com $34 \%$.

Palavras-chave: Encaminhamento, contrareferência, risco, cuidado e gravidez 


\section{Introducción}

Rosa Pryscilia Cardenas Urrelo

La mortalidad materna en el Perú ha sido un problema sanitario álgido llegado a registrar 93 x 100 mil nacidos vivos, en base a proyecciones del año 2015, donde urgía presentar cierta mejora en la mortalidad para acercarse a la meta de los Objetivos de Desarrollo del Milenio del Ministerio de Salud, establecida en 66 x 100 mil nacidos vivos (OMS, 2005).

Sin embargo, la mortalidad materna en el Perú para el año 2010 fue de 457 muertes, reduciéndose para el año 2014 a 411 muertes maternas, con estos reportes solo presento una reducción de un 10\% según la Dirección de Epidemiología del Ministerio de Salud (Minsa, 2015). En tanto, en el año 2016 se produjeron 415 muerte materna (MM) manteniéndose en índices inmejorables, apreciando un incremento de 4 muertes materna. (Minsa, 2016).

La mortalidad materna es uno de los indicadores de desarrollo de un país, siendo medido por el sistema sanitario, que tiene como propósito disminuir la mortalidad materna para alcanzar los objetivos, actualmente Objetivo de Desarrollo Sostenible (ODS) planteado por la Organización de las Naciones Unidas, donde su prioridad es reducir las causas de la mortalidad materna, partiendo de la identificación de los signos de alarma o factores de riesgo, los mismos que retrasan la toma de decisiones (proveedor - familiar) para el traslado del paciente a un establecimiento de salud que tenga la capacidad resolutiva para responder a la necesidad obstétrica y ser resuelto la emergencia. Sin embargo, en algunos casos el establecimiento de contacto que tiene el paciente es para estabilizar y realizar los primeros procesos de atención de acuerdo a su nivel de atención, luego poder ser referido a un establecimiento de mayor complejidad; es decir, haciendo del sistema de Referencia y Contrarreferencia. (Minsa, 2004).

A pesar de la implementación del sistema de referencia y contrarreferencia en la Dirección Regional de Salud de San Martín, no ha tenido resultados alentadores e incluso fuera de las proyecciones sanitarias; es así, que la Región San Martín en el año 2016 se produjeron solo $8 \mathrm{MM}$, el mismo que marco un gran hito en el sistema sanitario regional, respecto a los años 2015 y 2016 donde se produjeron 16 y 14 muertes maternas respectivamente. No obstante, para el año 2017 específicamente hasta la semana epidemiológica 52 se suscitaron $18 \mathrm{MM}$, el mismo que representa incremento superior al 100\% en comparación del año 2016 con apenas 8 MM. (Minsa, 2017).

Para el 2018, se registran aumentos muy significativos de muertes maternas de acuerdo al registro de ocurrencia fueron: Lima Metropolitana (+6), Áncash (+5), Callao 
(+4), Lima Provincias (+4) y Ucayali (+4). De lo contrario los departamentos que presentaron menos números de muertes en relación al mismo tiempo del 2017 fueron San Martín (-13), Cajamarca (-11), Piura (-6), Loreto (-6) y Cusco (-6) 34. En ese sentido existió una disminución considerable de MM en la región San Martín.

En ese sentido, la realidad regional de la muerte materna, expuesta en el párrafo anterior, surge la necesidad conocer el sistema de referencia y contrarreferencia en el Hospital Rural de Lamas, dicho establecimiento es el de mayor complejidad de la Provincia de Lamas, convirtiéndose en el centro de referencias de las micro redes de salud Caynarachi, Cuñumbuque, Rumizapa, Pacayzapa, Tabalosos y Lamas - Huayco que en su conjunto forman la Red de Salud Lamas. En la red de salud Lamas el sistema de referencia y contrareferencia comienza en los centros de servicios de salud de primer nivel de atención, quienes refieren a sus pacientes a centros de mayor complejidad, correspondiendo a las cabeceras de las micro redes de salud, y son las micro redes que trasladan a los pacientes críticos, los mismos que necesitan una atención especializado y son justamente que llegan derivados al Hospital Rural de Lamas que tiene categoría II-E.

Por todo lo anterior, el objetivo general de la presente fue determinar el nivel de cumplimiento de la norma técnica de referencias y contrarreferencias de las emergencias obstétricas en el Hospital Rural de Lamas.

\section{Metodología}

La presente investigación presenta un tipo de estudio no experimental con un diseño descriptivo simple, por cuanto se detallará las definiciones y características de las variables en estudio, en ese sentido se procederá a determinar la relación entre las mismas, a fin de resolver los objetivos propuestos y llegar a las conclusiones correctas (Hernández, 2010).

$\mathrm{M}-\mathrm{O}$

Donde

$\mathrm{M}=$ Muestra el nivel de cumplimiento de la norma técnica de referencias $\mathrm{y}$ contrarreferencias en el manejo las emergencias obstétricas en el Hospital Rural de Lamas $\mathrm{O}=$ Manejo las emergencias obstétricas en el Hospital Rural de Lamas 
La población de estudio estuvo constituida por todas las referencias de las gestantes, que hacen un total de 157, las mismas que fueron atendidas en el Hospital Rural de Lamas, en el periodo 2018.

La muestra de estudio estuvo constituida por el total de las referencias a las gestantes, las mismas que fueron desde las cabeceras de micro redes de salud al Hospital Rural de Lamas, constituida por 69 referencias obstétricas que fueron atendidas y referidas en el en el periodo 2018; cabe precisar que la muestra obtenida para el estudio se ajustó a los criterios de inclusión y exclusión, con la finalidad de encontrar homogeneidad y representatividad.

Seguidamente se presenta los criterios de inclusión y exclusión.

- Gestantes que fueron referidas al Hospital Rural de Lamas

- Gestantes con diagnóstico de emergencia obstétrica

- Gestantes referidas de las cabeceras de micro redes

- Gestantes referidas por personal de salud

En tanto, los Criterios de exclusión fueron:

- Gestantes con diagnóstico que no corresponden a una emergencia obstétrica

- Gestantes atendidas en el Hospital Rural de Lamas

- Gestantes residentes de la ciudad de Lamas

En la presente investigación se aplicó como técnica el análisis documental y la encuesta; siendo los instrumentos aplicados la guía de análisis documental y el cuestionario, con el propósito de recabar la información de los documentos sobre la referencia y contrarreferencia y la emergencia obstétrica en el Hospital Rural de Lamas.

El instrumento para determinar el nivel de referencia y contrarreferencia. El objetivo del instrumento fue determinar el nivel de manejo de referencia de pacientes obstétricas referidas al Hospital Rural de Lamas. (Tena, 2014).

El instrumento estuvo conformado por dos dimensiones, siendo la dimensión administrativa y asistencial; estas se agrupan en 16 items que fueron evaluados en el instrumento de referencia y contrarreferencia. Asimismo, la evaluación de los ítems fue de muy malo con puntaje 1 , el nivel malo con puntaje 2, regular con un puntaje de 3 , bueno con un puntaje de 4 , y muy bueno con un puntaje de 5 . 
Por su parte, el instrumento empleado en la medición de la emergencia obstétrica estuvo hecho en base al instrumento de Palomino y Ramos (2016). El objetivo del instrumento es conocer los diagnósticos más frecuentes con los que las mujeres embarazadas son referidas al Hospital Rural de Lamas.

El instrumento estuvo conformado por una sola dimensión, el mismo hace un total de 10 items que serán evaluados en el instrumento de emergencia obstétrica. Respecto a la medición de la variable, solo su propuso dos opciones, si cumple o no cumple; siendo valoraciones 0 y 1 .

El instrumento empleado en el estudio fue utilizado en investigaciones anteriores, siendo validadas con anterioridad a su aplicación por juicio de expertos.

En cuanto a la confiabilidad se aplicó la Escala Alfa de Cronbach cuyo valor debe ser mayores a 0.70 ; con ello indicar que los instrumentos son confiables para su aplicación.

Después de la recolección de datos, mediante los instrumentos dirigidos a la muestra de la investigación y luego de validar dichos instrumentos por los expertos se realizó un análisis sobre los resultados obtenidos, mediante el trabajo de campo en el desarrollo de investigación, con la finalidad de que la información obtenida sea coherente en función del problema de investigación, objetivos e hipótesis. Además, para el procesamiento de datos se utilizó medios electrónicos, calificados y sistematizados de acuerdo a la unidad de análisis, respecto a sus variables. El programa empleado fue el estadístico SPSS 24.

\section{Resultados y discusión}

En la tabla 1, se muestra el nivel de manejo administrativo de las referencias y contrarreferencias de pacientes obstétricas de los establecimientos de salud periféricos al Hospital Rural de Lamas, donde el nivel "Regular" de manejo administrativo alcanza un $33 \%$ (23), siendo este el nivel de mayor predominancia, seguido del nivel "Bueno" de manejo administrativo con un 25\% (17); asimismo, el nivel "Muy bueno" de manejo administrativo es representado con un 16\% (11), de la misma manera el nivel "Malo" de manejo administrativo corresponde a un 14\% (10) y el nivel "Muy malo" de manejo administrativo de las referencias y contrarreferencias alcanza un $12 \%$ respectivamente. 
Tabla 1.

Nivel de manejo administrativo de referencia y contrarreferencia de pacientes obstétricas desde los establecimientos de salud al Hospital Rural de Lamas

\begin{tabular}{lccc}
\hline \multicolumn{1}{c}{ Nivel } & Intervalo & $\mathbf{N}^{\circ}$ & $\mathbf{\%}$ \\
\hline Muy malo & $8-14$ & 8 & $12 \%$ \\
Malo & $15-20$ & 10 & $14 \%$ \\
Regular & $21-26$ & 23 & $33 \%$ \\
Bueno & $27-32$ & 17 & $25 \%$ \\
Muy bueno & $33-40$ & 11 & $16 \%$ \\
\hline \multicolumn{2}{c}{ TOTAL } & $\mathbf{6 9}$ & $\mathbf{1 0 0 \%}$ \\
\hline
\end{tabular}

En la tabla 2 se muestra el nivel de manejo asistencial de referencia y contrarreferencia de las pacientes obstétricas desde los establecimientos de salud al Hospital Rural de Lamas; donde el nivel "Regular" de manejo asistencial alcanza un 34\% (24), siendo este el de mayor predominancia, seguido del nivel "Bueno" de manejo asistencial con un 28\% (19); asimismo, el nivel "Muy bueno" de manejo asistencial alcanza un 17\% (12); en tanto, el nivel "Malo" y el "Muy malo" de manejo asistencial alcanzan un $12 \%(8)$ y $9 \%(6)$ respectivamente.

Tabla 2.

Nivel de manejo asistencial de referencia y contrarreferencia de pacientes obstétricas desde los establecimientos de salud al Hospital Rural de Lamas

\begin{tabular}{lccc}
\hline \multicolumn{1}{c}{ Nivel } & Intervalo & $\mathbf{N}^{\circ}$ & $\mathbf{\%}$ \\
\hline Muy malo & $8-14$ & 6 & $9 \%$ \\
Malo & $15-20$ & 8 & $12 \%$ \\
Regular & $21-26$ & 24 & $34 \%$ \\
Bueno & $27-32$ & 19 & $28 \%$ \\
Muy & $33-40$ & 12 & $17 \%$ \\
Bueno & TOTAL & $\mathbf{6 9}$ & $\mathbf{1 0 0 \%}$ \\
\hline
\end{tabular}

En la tabla 3 se muestra los diagnósticos más frecuentes con que son referidas las mujeres embarazadas al Hospital Rural de Lamas; donde el 29\% (20) de referencia tiene diagnóstico de "Aborto incompleto"; seguido de la hipertensión severa inducida por el embrazo en un $22 \%$ y otro de los diagnostico que destaca es el parto distócico o complicaciones. Por otro lado, los desgarros de cuello uterino y vaginal de III y IV grado tienen los menores valores porcentuales de referencia. 
Tabla 3.

Diagnósticos más frecuentes con que son referidas las mujeres embarazadas al Hospital Rural de Lamas

\begin{tabular}{lcc}
\hline \multicolumn{1}{c}{ Diagnóstico } & $\mathbf{N}^{\circ}$ & $\%$ \\
\hline Parto distócico o complicado & 11 & $16 \%$ \\
Recién nacido con complicaciones & 3 & $4 \%$ \\
Retención de placenta complicada & 6 & $9 \%$ \\
Hipertensión severa inducida por el embarazo & 15 & $22 \%$ \\
Hemorragia severa y shock hipovolémico & 7 & $10 \%$ \\
Sepsis Materna o Neonatal & 4 & $6 \%$ \\
Aborto incomplete & 20 & $29 \%$ \\
Desgarro vaginal grado III y grado IV & 2 & $3 \%$ \\
Desgarro de cuello uterino & 1 & $1 \%$ \\
\hline \multicolumn{1}{c}{ TOTAL } & $\mathbf{6 9}$ & $100 \%$ \\
\hline
\end{tabular}

En la tabla 4; se muestra el porcentaje de referencia realizadas por el personal de salud, siendo el profesional Obstetra que realiza el mayor porcentaje de referencias que corresponde a un 39\% (27), seguido del personal médico con un 25\% (17), cabe resaltar que también el personal técnico tiene un 13\% (9) de referencias, haciendo de ver que en algunos establecimientos no existe equipo completo de profesionales para brindar una atención a la gestante. De la misma manera el profesional de enfermería tiene una gran presencia en las referencias de las pacientes, correspondiendo a 23\% (16) respectivamente.

Tabla 4. Porcentaje de referencia realizadas por el personal de salud

\begin{tabular}{lcc}
\hline \multicolumn{1}{c}{$\mathbf{c}$} & $\mathbf{N}^{\circ}$ & $\mathbf{\%}$ \\
\hline Médico & 17 & $25 \%$ \\
Obstetra & 27 & $39 \%$ \\
Licenciado en Enfermería & 16 & $23 \%$ \\
Técnico de Enfermería & 9 & $13 \%$ \\
\hline \multicolumn{1}{c}{ TOTAL } & $\mathbf{6 9}$ & $\mathbf{1 0 0 \%}$ \\
\hline
\end{tabular}

En la tabla 5 se muestra el nivel de cumplimiento de la norma técnica de referencias y contrarreferencias de las emergencias obstétricas en el Hospital Rural de Lamas, donde el nivel "Regular" de cumplimiento de la norma técnica de referencias y contrarreferencias alcanza un 34\% (23), seguido del nivel "Bueno" de cumplimiento de la norma técnica de referencias y contrarreferencias con un $26 \%(18)$ y un $17 \%$ (12) de nivel "Muy bueno" de cumplimiento de la norma técnica de referencias y contrarreferencias; asimismo, el nivel "Malo" y "Muy malo" de cumplimiento de la 
norma técnica de referencias y contrarreferencias alcanza un $13 \%(9)$ y $10 \%(7)$ respectivamente.

Tabla 5.

Nivel de cumplimiento de la norma técnica de referencias y contrarreferencias de las emergencias obstétricas en el Hospital Rural de Lamas

\begin{tabular}{lccc}
\hline \multicolumn{1}{c}{ Nivel } & Intervalo & $\mathbf{N}^{\circ}$ & $\mathbf{\%}$ \\
\hline Muy malo & $8-14$ & 7 & $10 \%$ \\
Malo & $15-20$ & 9 & $13 \%$ \\
Regular & $21-26$ & 23 & $34 \%$ \\
Bueno & $27-32$ & 18 & $26 \%$ \\
Muy Bueno & $33-40$ & 12 & $17 \%$ \\
\hline & TOTAL & $\mathbf{6 9}$ & $\mathbf{1 0 0 \%}$ \\
\hline
\end{tabular}

Entre los principales hallazgos del estudio se muestra el nivel de cumplimiento de la norma técnica de referencias y contrarreferencias de las emergencias obstétricas en el Hospital Rural de Lamas, muestra el nivel "Regular" con un 34\%, seguido del nivel "Bueno" con un 26\% y un 17\% de nivel "Muy bueno"; asimismo, el nivel "Malo" y "Muy malo" alcanza un 13\% y $10 \%$ del cumplimiento de la norma técnica de referencias y contrarreferencias de las emergencias obstétricas en el Hospital Rural de Lamas.

Al contrastar con el estudio de Luo (2014) denominado "Sistema de salud en México: Un estudio de referencia y contrarreferencia en la atención médica en Hospital de segundo Nivel en el Distrito Federal - Hospital General Dr. Manuel Gea González"; donde concluyo que en México el sistema no funciona correctamente la parte de la referencia, y en cuanto a la contrarreferencia su existencia es prácticamente nula. Pudiendo inferir que en ambos estudios se encuentra similitud, en el caso de México el sistema de referencia y contrarreferencia no funciona adecuadamente, de la misma manera el estudio solo alcanza a un nivel regular, haciendo que aún carece de efectiva en el manejo y cumplimiento de la norma de referencia y contrarreferencia. En ese sentido, es necesario realizar una correcta estructuración y operación del sistema de referencia y contrarreferencia para garantizar una atención integral, oportuna y de optimización de recursos, con un enfoque que vincule la universalización de la salud.

Por su parte, Collado y Sánchez (2014) en su estudio titulado ¿Referencia y contrarreferencia o multi-rechazo hospitalario? Un abordaje cualitativo; donde concluyo 
que el $57 \%$ de las mujeres tuvieron que buscar más de un sitio para su resolución obstétrica: $25 \%$ visitó dos, $17 \%$ tres, $15 \%$ a más de tres. La mujer que más hospitales visitó, acudió a nueve nosocomios a lo largo de 24 horas. Al contrastar con el estudio, muestra que los diagnósticos más frecuentes con que son referidas las mujeres embarazadas al Hospital Rural de Lamas, es el "Aborto incompleto" con un 29\% del total de las referencias, seguido de la hipertensión severa inducida por el embrazo en un $22 \%$.

En la comparación se puede inferir que las condiciones donde se desarrolla el sistema de referencia y contrarreferencia representan un obstáculo para el acceso a la atención obstétrica hospitalaria oportuna. Siendo necesario fortalecer el sistema para asegurar la absolución de los casos para evitar los rechazos que tiene repercusiones negativas en el manejo de la gestante y finalmente en la salud de las mujeres.

De la misma manera en su estudio de Palomino y Ramos (2016) titulado "Manejo de las referencias y contrarreferencias de las pacientes obstétricas atendidas en el Centro de Salud de Anta"; donde concluyo que un 36\% las pacientes son referidas por Hipertensión Inducia por la Gestación, por lo que en un $79 \%$ son referidas hacia emergencia. Con respecto a la contrarreferencia se obtuvo que en el $70 \%$ no lleguen los formatos hacia el Centro de Salud. Asimismo, el estudio hace mención que no existe un buen manejo de referencias y contrarreferencias en el Centro de Salud de Anta.

Al contrastar con el estudio de Palomino y Ramos (2016) el estudio muestra que el "Aborto incompleto" con un 29\% del total de las referencias, seguido de la hipertensión severa inducida por el embrazo en un $22 \%$; existiendo similitud con el estudio en mención. Asimismo, el estudio muestra el nivel "Regular" con un 34\%, del cumplimiento de la norma técnica de referencias y contrarreferencias de las emergencias obstétricas en el Hospital Rural de Lamas; esta última muestra divergencia.

\section{Conclusiones}

- El nivel de cumplimiento de la norma técnica de referencias y contrarreferencias de las emergencias obstétricas en el Hospital Rural de Lamas, muestra el nivel "Regular" con un 34\%, seguido del nivel "Bueno" con un 26\% y un 17\% de nivel "Muy bueno"; asimismo, el nivel "Malo" y "Muy malo" alcanza un 13\% y 10\% del cumplimiento de la norma técnica de referencias y contrarreferencias de las emergencias obstétricas en el Hospital Rural de Lamas.

- El nivel de manejo administrativo de las referencias y contrarreferencias de pacientes obstétricas de los establecimientos de salud periféricos al Hospital Rural 
de Lamas, muestra un nivel "Regular" de un 33\%, seguido del nivel "Bueno" con un 25\%; asimismo, el nivel "Muy bueno" es representado con un 16\%, de la misma manera el nivel "Malo" corresponde a un 14\% y el nivel "Muy malo" alcanza un $12 \%$ de manejo administrativo de las referencias y contrarreferencias, siendo este el nivel "Regular" el de mayor predominancia en el manejo administrativo.

- El nivel de manejo asistencial de referencia y contrarreferencia de las pacientes obstétricas desde los establecimientos de salud al Hospital Rural de Lamas; muestra un nivel "Regular" con un 34\%, seguido del nivel "Bueno" con un 28\%; asimismo, el nivel "Muy bueno" alcanza un 17\%; en tanto, el nivel "Malo" y el "Muy malo" alcanzan un 12\% y 9\% de manejo asistencial de referencia y contrarreferencia de las pacientes obstétricas; siendo este el nivel "Regular" el de mayor predominancia en el manejo asistencial.

- Los diagnósticos más frecuentes con que son referidas las mujeres embarazadas al Hospital Rural de Lamas, es el "Aborto incompleto" con un 29\% del total de las referencias, seguido de la hipertensión severa inducida por el embrazo en un $22 \%$; en tanto, los desgarros de cuello uterino y vaginal de III y IV grado tienen los menores valores porcentuales de los diagnósticos que fueron causa de referencia.

- El porcentaje de referencia realizadas por el personal de salud, siendo el profesional Obstetra con el mayor porcentaje de referencias correspondiente a un $39 \%$, seguido del personal médico con un $25 \%$, de la misma manera el profesional de enfermería le corresponde un $23 \%$ de referencias, resaltando que el personal técnico tiene un $13 \%$ de referencias, mostrando que en algunos establecimientos existe la dificultad de construir los equipos de atención para la atención a la gestante.

\section{Referencias bibliográficas}

Collado, S. P., \& Sánchez, Á. (2014) ¿Referencia y contrarreferencia o multi-rechazo hospitalario? Un abordaje cualitativo. [Tesis maestría]. México D.F: Universidad Autónoma Metropolitana.

Hernández, R. (2010) Metodología de la Investigación. Lima: Mc Graw Hill.

Luo, L. (2014) Sistema de salud en México: Un estudio de referencia y contrarreferencia en la atención médica en Hospital de segundo Nivel en el Distrito Federal 
"Hospital General Dr. Manuel Gea González". [Tesis maestría]. México D. F: Universidad Nacional Autónoma de México.

MINSA (2004). RM N $N^{\circ}$ 751. Norma Técnica del Sistema de Referencia y Contrareferencia de los Establecimientos del Ministerio de Salud. Lima.

MINSA (2015). Reducción de la mortalidad materna [en línea]. URL disponible en: www.minsa.gob.pe/?op $=51 \&$ nota $=16498$

MINSA (2016) Indicadores trazadores: mortalidad materna; 2016. [en línea]. URL disponible en http://www.minsa.gob.pe/?op=6

MINSA (2017). Centro Nacional de Epidemiologia, Prevención y Control de Enfermedades. Muerte Materna según departamento de ocurrencia y procedencia Perú. Lima.

OMS (2005). Informe sobre la salud en el mundo: ¡cada madre y cada niño contarán! Ginebra: OMS.

Palomino, M. C., \& Ramos, J. S. (2016) Manejo de las referencias y contrarreferencias de las pacientes obstetricas atendidas en el Centro de Salud de Anta. Cusco. [Tesis maestría]. Universidad Andina del Cusco, Perú.

Tena, A. F. (2014). Causas de referencia para resolución obstétricia en mujeres embarazadas atendidas en el Centro Especializado de Atención Primaria a la salud de San Miguel Chapultepec Bicentenerio. Toluca. [Tesis maestría]. Universidad Autónoma del Estado de México. 